\title{
ANGULAR MOMENTUM TRANSPORT IN ACCRETION DISKS: A HYDRODYNAMICAL PERSPECTIVE
}

\author{
S. Fromang ${ }^{1}$ and G. Lesur ${ }^{2}$
}

\begin{abstract}
The radial transport of angular momentum in accretion disk is a fundamental process in the universe. It governs the dynamical evolution of accretion disks and has implications for various issues ranging from the formation of planets to the growth of supermassive black holes. While the importance of magnetic fields for this problem has long been demonstrated, the existence of a source of transport solely hydrodynamical in nature has proven more difficult to establish and to quantify. In recent years, a combination of results coming from experiments, theoretical work and numerical simulations has dramatically improved our understanding of hydrodynamically mediated angular momentum transport in accretion disk. Here, based on these recent developments, we review the hydrodynamical processes that might contribute to transporting angular momentum radially in accretion disks and highlight the many questions that are still to be answered.
\end{abstract}

\section{Introduction}

One of the many research activities of Jean Paul Zahn during his career was devoted to studying the mechanisms responsible for outward angular momentum transport in accretion disks. More specifically, he focused on the possibility that hydrodynamical turbulence could develop and lead to enhanced and efficient transport: after all, accretion disks are shear flows, and our experience of shear flows in the laboratory and in our every day life is that they are easily destabilized and prone to turbulence. As we shall see, this turned out to be much more complicated than anticipated, to the point that the very existence of such a hydrodynamic transition to turbulence in accretion disks is still vividly debated today and far from being settled. Nevertheless, Jean-Paul's approach to the problem reinvigorated the interest of the astrophysical community for hydrodynamic (as opposed

${ }^{1}$ Laboratoire AIM, CEA/DSM-CNRS-Université Paris 7, Irfu/Service d'Astrophysique, CEA-Saclay, 91191 Gif-sur-Yvette, France

2 Univ. Grenoble Alpes, CNRS, IPAG, 38000 Grenoble, France 
to magneto-hydrodynamic) processes in accretion disks in general. This renewed interest led to unexpected discoveries in the past few years, and it is now clear that hydrodynamics processes are important if we want to reach a complete and coherent picture of accretion disks dynamics. The aim of this review is to provide the community with a description of these recent advances.

Before doing so, we start by recalling the problem at hand. Accretion disks are objects of disk-like shape, mostly composed of gas rotating around a central object of mass $M$. In this review, we will restrict the discussion to situations for which collisions between particles are frequent enough that the dynamics can be described by the hydrodynamics equations, although we note that this is not always the case, as for example in the inner parts of disks rotating around supermassive black holes (Quataert 2001). Observations of accretion disks indicate that the disk material falls on the central object. This is true for accretion disks of all natures, regardless of whether the central object is a supermassive black hole, a stellar mass compact object or a young star (Frank et al. 1985). It is precisely the existence of that accretion flow that is difficult to understand theoretically because of angular momentum conservation: a particle with a given angular momentum $\mathcal{L}$ with respect to the central object tends to settle into a circular orbit around that object with a Keplerian angular frequency $\Omega_{K}$ such that gravitational attraction is balanced by the outwardly directed centrifugal force. For this particle to fall onto the central object, it must loose its angular momentum. Finding viable and efficient mechanisms for doing so has been the subject of intense research activities in the past 50 years. Broadly speaking, one can distinguish between two types of phenomena. Internal processes creates an outwardly directed flux of angular momentum in the bulk of the disk, for example in the form of turbulence or waves, whereas external processes extract angular momentum from the disk by an external torque. The later possibility encompasses disks winds and jets that might be associated with a large scale magnetic field for example (Frank et al. 2014). In this review, we will focus on the former, and only discuss occasionally the connection that might exist between both.

The processes operating within the disk can themselves also be separated in two classes, depending on whether or not they depend on the presence of a magnetic field. This review is devoted to processes that operate in disk in the absence of a magnetic field. However, the field has been dominated in the past thirty years by research connected to the so-called magneto-rotational instability (MRI, Balbus \& Hawley 1991, 1998), to the point that many readers may wonder whether it is still even worth considering hydrodynamic processes at all. For this reason, we start in Section 2 by discussing the difficulties that remain associated with the MRI paradigm, but also take the opportunity to quickly review the latest results in that field, some of which are relevant for the present review. In Section 3, we go back to the pure hydrodynamic case and briefly discuss the linear stability of accretion disks in this context. We next focus in Section 4 on the possible existence of a subcritical transition to turbulence in these objects, highlighting the contribution of Jean-Paul Zahn in this field. As we shall see, the question is not yet settled, and several researchers have gone to explore other routes in the past 
few years. In particular, much attention has been devoted, with some success, to the consequences of the peculiar thermodynamics of protoplanetary disks. We thus describe the most promising of these results in Section 5. Finally, we briefly discuss in Section 6 some of the many other possibilities that have been proposed or are currently being debated in the literature to account for angular momentum transport in accretion disks.

\section{The magnetorotational instability and its shortcomings}

The question of the potential relevance of hydrodynamics effects in disks may be deemed irrelevant because we know there exists a powerful magnetohydrodynamical (MHD) instability in accretion disks: indeed, flows in keplerian rotation around a central object are destabilized by a weak magnetic field. Although known since the 1960's (Velikhov 1959; Chandrasekhar 1961); the relevance of that instability for accretion disks was only realized three decades later by (Balbus \& Hawley 1991). The discovery of the magneto-rotational instability (MRI), as it was to be called, revolutionized our understanding of angular momentum transport in accretion disks. This is because the MRI grows on dynamical timescales, has minimal geometrical requirements (a subthermal B-field and a radially decreasing angular velocity) and leads during its nonlinear evolution to fully developed MHD turbulence and an associated outward flux of angular momentum that is roughly (i.e. to within an order of magnitude) compatible with observational constraints (Balbus \& Hawley 1998). At first glance, it appears that the MRI is the ideal solution to angular momentum transport in accretion disks.

However, the MRI paradigm is not without problems. Indeed, in the cold and dense protoplanetary $(\mathrm{PP})$ disks, the ionization fraction is so small that the gas and the field are not well coupled: ideal MHD does not apply and non-ideal effects cannot be ignored. Considering the stabilizing effects of ohmic resistivity on the linear instability, Gammie (1996) first showed that many regions of PP disks are immune to the MRI: accretion proceeds only through the disk upper layers thanks to MRI-driven MHD turbulence, while their mid-plane remains laminar. This layered accretion scenario was later confirmed using numerical simulations (Fleming \& Stone 2003) and the dead zone paradigm became the dominant and widely accepted scenario during the following decade. However, it only includes the effect of Ohmic diffusion, whereas typical densities and temperatures expected in PP disks show that both ambipolar diffusion and the Hall effect should be dominant in most parts of these objects. Both processes turned out to affect the conclusions of Gammie (1996). The addition of ambipolar diffusion in the layered accretion paradigm leads to an even more dramatic picture where MRI turbulence is severely suppressed in most of the disk, leading to accretion rates at least 10 times lower than observed ones (see Turner et al. 2014, for a review). In order to "save the MRI", magnetised disk winds, mostly neglected since the discovery of the MRI, came back to the scene as a viable alternative to MRI turbulence. Effectively, the MRI naturally produces magnetized outflows upon saturation provided that the disk is threaded by an external poloidal field (Suzuki \& Inutsuka 
2009; Suzuki et al. 2010; Bai \& Stone 2013; Fromang et al. 2013). This wind, launched from the very surface of the disk ionised by far-UV radiations, could be sufficient to recover accretion rates compatible with observations (Bai 2016). The addition of the Hall effect leads to an even more complicated picture since it is a purely dispersive effect, which does not lead to energy dissipation. It induces significant modifications to the flow topology, including the appearance of strong zonal flows (Kunz \& Lesur 2013) and/or an increased importance of disk winds in torquing the disk (Lesur et al. 2014; Bai 2014, 2015). Many aspects of Hall mediated accretion remain to be elucidated, largely because these results have been obtained in a local framework and may suffer from undesired but uncontrolled artifacts. Nevertheless, at the time this review is being written, we cannot rule out the possibility that angular momentum transport in PP disks is regulated by processes at least partially governed by Hall physics.

Given all these uncertainties, it is very tempting to consider alternative routes to angular momentum transport that do not rely on MHD processes. This motivated several groups, including Jean-Paul Zahn's, to investigate purely hydrodynamical instabilities.

\section{The hydrodynamical stability of accretion disks}

In cylindrical coordinates $(R, \phi, z)$, the stability of non-magnetized rotating sheared flows is well described by the Solberg-Høiland criterion, which is a sufficient condition for stability under the action of infinitesimal axisymmetric and adiabatic perturbations (Tassoul 2007):

$$
\frac{1}{R^{3}} \frac{\partial j^{2}}{\partial R}-\frac{1}{C_{p} \rho} \nabla P \cdot \nabla \boldsymbol{S}>0
$$

and

$$
\frac{\partial P}{\partial z}\left(\frac{\partial j^{2}}{\partial R} \frac{\partial S}{\partial z}-\frac{\partial j^{2}}{\partial z} \frac{\partial S}{\partial R}\right)<0,
$$

where $j$ is the specific angular momentum, $C_{p}$ the calorific capacity at constant pressure, $\rho$ the density, $P$ the pressure and $S$ the entropy of the gas under consideration.

To illustrate how this criterion applies to accretion disks, let us consider a simple accretion disk model with a Keplerian rotation profile

$$
\Omega_{K}=\sqrt{\frac{G M}{R^{3}}} .
$$

The first Solberg-Hoilland criterion (3.1) can be recast as

$$
\Omega_{K}^{2}+N_{R}^{2}>0
$$

where we have introduced the radial Brunt-Väissälä frequency $N_{R}$

$$
N_{R}^{2}=-\frac{1}{\gamma \rho} \frac{\partial P}{\partial R} \frac{\partial}{\partial R} \ln \left(\frac{P}{\rho^{\gamma}}\right) .
$$


In thin disks, where the disk thickness $H$ is much smaller than the disk radius $R$, we have $\left|N_{R}^{2}\right| \sim(H / R)^{2}$, so that for all practical purposes, this criterion is always satisfied. In the case where radial buoyancy vanishes (and hence entropy is constant over radius), we recover the usual Rayleigh criterion which predicts stability for flows with increasing specific angular momentum $j$.

In this simple disk model, the rotation profile $\Omega_{K}$ only depends on $R$. For this reason, the second Solberg-Hoilland criterion (3.2) reduces to the Schwarzschild criterion for vertical convection:

$$
N_{z}^{2}>0
$$

with the vertical Brunt-Väissälä frequency $N_{z}^{2}$. This criterion is usually satisfied in $\mathrm{PP}$ disks where the disk surface is hotter than the disk mid-plane due to external irradiation. However, some disks heated by turbulent dissipation sometime violate this criterion, especially when the gas reaches very high opacities, such as in dwarf novae, and convection might develop (see Sect. 6).

\section{Subcritical transition to turbulence}

\subsection{The basic idea}

As shown above, the radial profile of angular velocity in accretion disks is Keplerian and is therefore stable to axisymmetric infinitesimal perturbations. However, linear axisymmetric stability is not necessarily the full story. There are many examples of linearly stable flows that become unstable and develop turbulence at large Reynolds numbers such as Poiseuille (pipe) flows and plane Couette flows. Such a transition requires finite amplitude (as opposed to vanishingly small) fluctuations and is called a subcritical transition. While linearly stable at all Reynolds number $R e$, turbulence is easily observed in plane Couette experiments whenever Re reaches $\sim 1600$ (Tillmark and Alfredsson 1992). The later is typically defined as

$$
R e=\frac{S L^{2}}{\nu}
$$

where $\nu$ stands for the fluid kinematic viscosity, while $S$ is the shear rate and $L$ is the distance between the walls. In accretion disks, order of magnitude estimates of $R e$ are fairly easy to obtain. For example, Frank et al. (1985) quote Re $\sim 10^{14}$ as being typical for disks around white dwarfs in binary systems. This is a general result: $R e$ is always larger by many orders of magnitudes than the typical critical Reynolds numbers above which turbulence is found for most linearly stable shear flows in the laboratory. These simple arguments led many researchers to conjecture that a nonlinear transition to turbulence might also exist in accretion disks and be responsible for outward angular momentum transport in these objects.

\subsection{Jean Paul Zahn's era}

Finding such a transition, however, proved more challenging than one might naively think. This is because of the Coriolis force. Compared to standard Couette 
flows, it promotes the occurrence of epicyclic motions and has a stabilizing influence on the flow (Balbus et al. 1996). In fact, early numerical simulations, performed in the framework of the so-called shearing box (Goldreich \& Lynden-Bell 1965), a well-known local model of accretion disk, failed to find any signature of turbulence in Keplerian flows (Balbus et al. 1996; Hawley et al. 1999). However, the limited spatial resolution that could be reached at that time, associated with the uncontrolled dissipation of the numerical method that was used, prevented definite conclusions to be drawn from these results.

This is when and why Jean-Paul Zahn started to work on the problem. Along with his collaborators and $\mathrm{PhD}$ students, he realized that fluid experiments could help improve our understanding of the problem. In this regard, the Taylor-Couette flow is particularly interesting: it consists in two concentric rotating cylinders sandwiching a fluid, which is generally an incompressible liquid. Drag exerted by the rotating cylinders onto the liquid sets the later in rotation. By varying the angular velocities of both cylinders, the angular velocity and angular momentum radial profile of the flow within the cavity and its Reynolds number can be varied. While the Taylor-Couette apparatus has been the subject of active research since the pioneering work of Taylor (1923), the regime closest to accretion disk had only been barely considered by the end of the $20^{\text {th }}$ century.

The contribution of Jean-Paul Zahn was twofold: first, along with his students, he re-analyzed results from old experiments performed in a regime of radially increasing angular momentum by Wendt (1933) and Taylor (1936). These old results hinted toward a transition to a chaotic flow, possibly turbulence, at large Reynolds number (Richard \& Zahn 1999). But Jean-Paul Zahn also fully realized the shortcomings of these conclusions, based on a series of old experiments. This is why he initiated a collaboration with a team of physicists at the CEA with whom he embarked into building his own Taylor-Couette experiment (see Fig. 1, left panel). Using this new device, the group studied, for the first time with modern techniques, the regime most analogous to accretion disks. While claiming, by visual inspection, a transition to turbulence, they also failed to properly characterized its properties (Richard 2001). In their conclusions, they noted that an improvement of the treatment of the ends caps of the experiment as well as extending the investigation toward higher Reynolds numbers (their largest $R e$ value is of the order of a few times $10^{4}$ ) were both needed. But the stage was set for future investigation. And in fact, Richard \& Zahn (1999) close their paper with the following sentence: "(...) we hope that experimentalists will turn again to this classical problem, which is of such great interest for geophysical and astrophysical fluid dynamics, and that they will explore the rotation regimes for which the data are so incomplete." As we shall see now, their hope has been largely fulfilled...

\subsection{The current state of affair}

Jean Paul's efforts to motivate the scientific community toward experimental astrophysics has been successful indeed: the regime of outwardly increasing angular momentum flows is now the subject of active research by the community of 

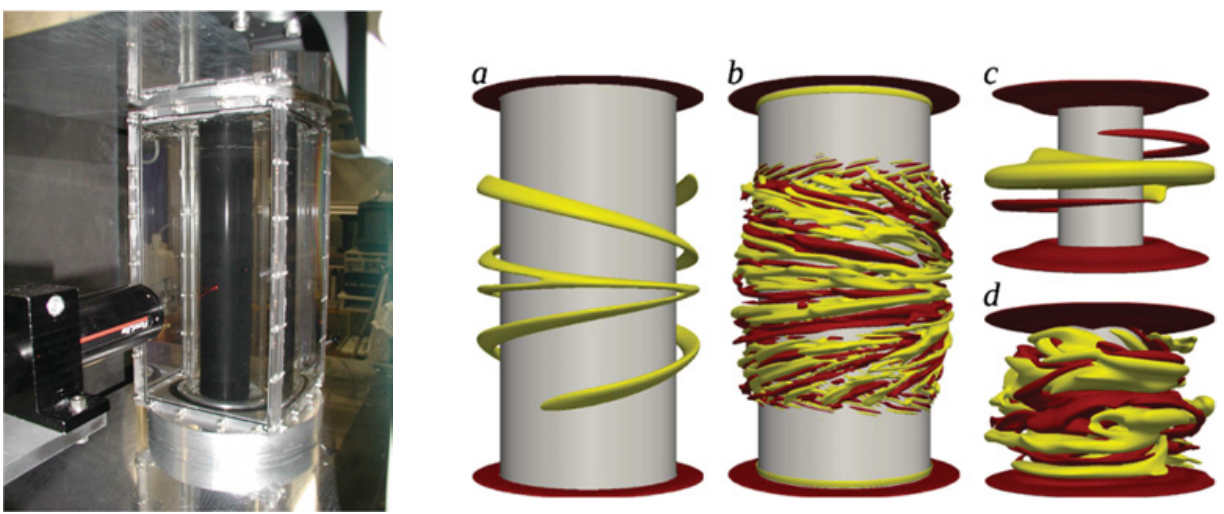

Fig. 1. Left: the CEA Taylor-Couette experiment built in collaboration with Jean-Paul Zahn in the early 2000's (Credit: CEA/DSM/IRAMIS/SPEC). Right: Isosurface of the radial velocity in simulations of the Maryland (panels a and b) and Princeton (panels c and d) experiments at $R e=1332$ (a), $R e=5328$ (b), $R e=1545$ (c) and $R e=6437$ (d). From Avila (2012).

experimentalists experts in Taylor-Couette flows. In that respect, the interested reader will benefit from the recent review of Grossmann et al. (2016).

What did we learn from this intense research activity? The least we can say is that the status of the field is confused! Some groups report that Keplerian flows become turbulent, some other that they remain laminar. The experiments that paid particular attention to the regime most relevant for accretion disks can be divided in two groups. On the one hand, the Twente (van Gils et al. 2011) and Maryland (Paoletti \& Lathrop 2011) experiments both report that the flow is turbulent. The claim is based on measurements of an increased torque exerted by the fluid on the rotating cylinders compared to what would be expected from a laminar flow. The results are consistent between the two experiments (Paoletti et al. 2012). On the other hand, the Princeton experiment (Ji et al. 2006; Schartman et al. 2012; Edlund \& Ji 2014) reports that the flow remains laminar up to a Reynolds number equal to $2 \times 10^{6}$, a value comparable in amplitude to that reached by both the Twente and Maryland experiments. The claim is based on doppler velocity measurements. In addition to confirming this result, Schartman et al. (2012) measured increased velocity fluctuations near the end caps of the Princeton apparatus (see their Figs. 4 and 7), which they suggest might arise because of an unstable shear layer that develops at that location. To our knowledge, there is no real understanding accepted in the community that would explain these conflicting results. The experiment devices have differences: the Twente and Maryland apparatus are tall and narrow (the height $L$ ranges between 70 and 90 centimeters and the width $\Delta R$ is of the order of $6 \mathrm{~cm}$ ), while the Princeton experiment is small and wide $(L=28 \mathrm{~cm}$ and $\Delta R \sim 13 \mathrm{~cm})$. Such a difference in the apparatus "curvature" (quantified as the ratio $\Delta R / R$ ) is known to significantly 
affect the critical Reynolds number of subcritical transitions (Richard \& Zahn 1999), making turbulence more difficult to reach in a wide apparatus. Using the scaling proposed by (Richard \& Zahn 1999), the critical Reynolds number of the Princeton experiment should be around $6 \times 10^{5}$ (Ji et al. 2006), i.e., very close to the maximum Reynolds number reached in this experiment (a few times $10^{6}$ ). Therefore, there is a possibility that the Princeton experiment is below or right at the critical Reynolds number of the transition due to the dimensions of the apparatus, leading to a laminar flow. Another difference lies in the treatment of the end caps of the experiments: both axial boundaries co-rotates with the outer cylinder in the Twente and Maryland experiments, while they are composed of two independently rotating rings in the Princeton experiments. In order to investigate how these differences might affect the flow, Avila (2012) performed dedicated direct numerical simulations of the two experiments. Of course, being limited by current computational capabilities, the maximum Reynolds number that could be reached in the simulations, of order a few thousands, is much smaller than that of the experiments. Nevertheless, the results show that the flow becomes turbulent in both simulations (see Fig. 1, right panel) and that this transition is due to the boundaries. This has been confirmed by recent simulations at Reynolds number up to $\sim 50000$ where turbulence was found to be more and more confined to the vicinity of the end caps as Re increases, while the bulk of the flow remains laminar (Lopez \& Avila 2017): finite size effects are definitely important in the experiments and should be investigated carefully.

In order to free themselves from such complications associated with the boundaries, but also to consider geometries that would be closer to actual accretion disks than Taylor-Couette experiments, a few groups performed numerical simulations that addressed the question of the subcritical transition to turbulence using different numerical setups. First, Lesur \& Longaretti (2005) revisited the results presented by Balbus et al. (1996) and Hawley et al. (1999). Performing direct numerical simulations in the framework of the shearing box, they tried to evaluate the evolution of the critical Reynold number $R e_{c}$ (above which the flow becomes turbulent) as one enters the Rayleigh stable regime. They concluded that $R e_{c}$ lies in the range $10^{10-26}$ for Keplerian flows and that such large critical Reynolds number would lead to angular momentum transport rates that would be, for all astrophysical purposes, much smaller than required by the observations. The weakness of their approach, though, lie in the massive extrapolation involved because only states very close to the Rayleigh stable line could be investigated numerically due to the massive computational requirements required when $R e$ grows above a few thousands. This explains the enormous range in the value of $R e_{c}$ quoted above. More recently, and benefiting from the modern computational resources that are now available, Ostilla-Mónico et al. (2014) investigated Taylor-Couette flows with periodic axial boundaries (so that they avoid the complexity associated with the end caps discussed above) for Reynolds numbers up to $\sim 10^{5}$. Their strategy consisted in starting from a turbulent flow (obtained in the Rayleigh unstable regime by choosing an outer cylinder at rest), and suddenly enter the stable regime by 
setting the outer cylinder in rotation. In all their simulations, Ostilla-Mónico et al. (2014) found that turbulence decays, although with longer and longer timescales as $R e$ goes up. Both of the aforementioned studies agree in the fact that $R e_{c}$ is larger than $10^{5}$.

Because of the finite computational ressources at our disposal and the gigantic Re of accretion disks, numerical simulations will always suffer from the limitations highlighted above. Alternative approaches are thus desirable. This is the purpose of methods based on the so-called linear transient growth approach, such as originally proposed for this problem, in collaboration with Jean-Paul Zahn, by Tevzadze et al. (2003), and further described in this volume by Gogichaishvili et al. (2017). As is well known, the linear operator describing rotating sheared flows is non-normal, so that linear perturbations can see their energy grow by several orders of magnitude for a limited amount of time before being ultimately dissipated. It has been suggested that these transient amplifications could be the route to turbulence in sheared flows since transiently amplified modes could become non-linear and excite new modes which could be amplified again, etc. The key question here is to know whether non-linear interactions can regenerate sufficiently rapidly new transient modes. It is therefore critical to understand how this non-linear feedback proceeds (or fails) in astrophysical flows, a point that still deserves to be established. We end by stressing that, while having transient amplification might be required for the flow to be subject to a subcritical transition to turbulence, it is clearly not a sufficient condition, as recently illustrated by Shi et al. (2017). For all these reasons, the importance of such transiently growing disturbances for angular momentum transport in accretion disks still remains to be firmly established. An alternative and maybe more promising approach is to focus on isolating steady and/or oscillating structures in the flow that would form the skeleton for the turbulent dynamics. Based on this idea, Rincon et al. (2007) used continuation methods to find steady nonlinear solutions for Rayleigh stable cyclonic flows (i.e., $d \Omega / d R>0$ ) but failed to do so for anticyclonic flows (and therefore Keplerian profiles), for which $d \Omega / d R<0$, suggesting the later were more difficult to destabilize by non-linear instabilities than the former. Although not fully conclusive, such an approach could serve as a starting point for future theoretical work.

More than 20 years after Jean-Paul Zahn first tried to address this problem, the question of whether or not accretion disks are vulnerable to a subcritical transition to turbulence cannot yet be unambiguously answered.

\section{The importance of the disk thermodynamics}

In real life, accretion disks are not composed of an incompressible liquid rotating between rigid cylinders! Some of them are irradiated by the central object around which they rotate, while all of them radiate away some of their internal energy into space. In other words, their thermal structure is governed by complex processes. In recent years, this has been shown to be important for their stability. The purpose 
of the present section is to discuss these recent results, which have mostly been obtained in the context of PP disks. In order to fix ideas, we start by recalling the properties of these objects and their peculiar thermal structure.

\subsection{Protoplanetary disk properties and thermal structure}

PP disks are found rotating around newly born stars. Thanks to the wealth of observations that are now available, we have a fairly good idea of their basic properties (see Williams \& Cieza 2011, for a review). They are mostly composed of gas, but also contain about $1 \%$ in mass of solid particles (usually refereed to as dust), which is very important for setting disks opacities. Incidentally, dust particles are also believed to be the building blocks of planets. Disk masses are very uncertain because they are mostly composed of optically thick hydrogen which is actually very difficult to see, but the estimates are of the order of a few percent of the central stellar mass. Their lifetime, also uncertain, is of the order of a few millions years.

To first order, PP disks are essentially passive disks: their thermal structure is set by a balance between heating due to the irradiation of the disk upper layers by the central star, mostly at UV and visible wavelengths, and cooling due to the dust particles thermally radiating their energy to space at infrared wavelengths (Chiang \& Goldreich 1997). Simple $1+1 \mathrm{D}$ models can be constructed and enable the $(R, Z)$ temperature distribution to be estimated with a fair degree of accuracy (see Dullemond et al. 2007, for a review). They show that the mid-plane temperature of PP disks decays with the distance $R$ to the central star (with a power law scaling close to $R^{-1 / 2}$ ). Its vertical profile is essentially isothermal outward of approximately a couple of astronomical units (AUs) in the bulk of the disks except for the very tenuous surface layers where it increases due to stellar irradiation. Internal heating, such as for example induced by turbulent dissipation, only starts playing a role in the few inner tens of AU (D'Alessio et al. 1998).

In recent years, these simple models have been extended to include more realistic treatments of the radiative transfer (opacity, geometry) and additional physical processes (dust and gas decoupling, chemistry). Perhaps the most realistic disks structure that have been published so far are the radiation chemico-dynamical models that can be calculated using the code PRODIMO (see Woitke et al. 2009, and the subsequent improvements of the method). They include dust continuum radiative transfer, gas-phase and photo-chemistry, as well as gas thermal balance, and compute the disks hydrostatic vertical structure assuming it is axisymmetric. Detailed calculations using PRODIMO roughly confirm the disk structure described above and can be summarized as follows: radially decreasing and relatively cold temperature in the mid-plane, sandwiched between hot surface layers where dust and gas temperatures decouple.

Disk temperatures, however, are not neccessarily constant and may vary with time: how important an effect it is depends on how fast the material can heat and cool. This effect can be evaluated by means of the cooling time $\tau_{c}$. In the midplane, cooling is mediated by dust particles and is efficient, so that $\tau_{c}$ is only a 
fraction of the orbital timescale (Woitke et al. 2009; Lin \& Youdin 2015; Kratter \& Lodato 2016). $\tau_{c}$ increases in the low-density upper layers of the disks where it is dominated by atomic lines cooling and becomes longer than the orbital timescale. For example, at $10 \mathrm{AU}, \mathrm{PRODIMO}$ calculations suggest that $\tau_{c} \sim 50$ to 100 years at locations a few scale heights above the disk mid-plane (Woitke et al. 2009, see their Fig. 13).

\subsection{The baroclinic instability (SBI) and the convective over-stability}

The ideas developed above lead to the realization that the radial thermal structure of PP disks may play a role in their dynamics. However, this route to instability is not straightforward since the linear stability criterion (3.4) shows that thin disks are linearly stable to adiabatic perturbation. The key here it to realize that perturbations are not necessarily adiabatic in PP disks, essentially because the cooling timescale can be of the order of the orbital timescale in the mid-plane (see discussion above). This fact alone is enough to violate the Solberg-Hoïland criterion, but it is not enough to make the flow unstable! Indeed, the baroclinic instability and the convective over-stability are both thermal instabilities. Their source of free energy is not the shear but the thermal structure of the flow. For this reason, in addition to non-adiabatic perturbations, they also require the Schwarzschild criterion for radial convection to be satisfied, i.e. $N_{R}^{2}<0$.

\subsubsection{The baroclinic instability (SBI)}

The baroclinic instability arises in $2 \mathrm{D}(R, \phi)$ razor-thin disks in the presence of nonaxisymmetric disturbances when the two conditions above are satisfied $\left(N_{R}^{2}<0\right.$, finite $\tau_{c}$ ). Early simulations by Klahr \& Bodenheimer (2003), later supported by numerical evidences from Petersen et al. (2007a, b), first reported the spontaneous growth and long term survival of vortices in this situation. It even appeared that the associated density fluctuations, driven by the coupling between incompressible vortices and sound waves (Heinemann \& Papaloizou 2009), were able to create a significant angular momentum flux (Klahr \& Bodenheimer 2003). However, some of these results were sensitive to the numerical properties of the algorithm (Johnson \& Gammie 2005) and the linear stability analysis, suggesting only transient amplification of linear disturbances (Klahr 2004; Johnson \& Gammie 2005), was difficult to interpret, so that the very existence of a baroclinic instability in PP disks was subject to caution. Later numerical results, however, helped clarify its existence, origin and properties: The baroclinic instability exists but it is, in fact, subcritical: finite amplitudes disturbances are necessary to reach a turbulent state (Lesur \& Papaloizou 2010). For this reason, the baroclinic instability is now refereed to as the subcritical baroclinic instability, or SBI for short. The subcritical nature of the instability explains the failures of the linear analysis mentioned above to unambiguously identify the mechanism at work. 


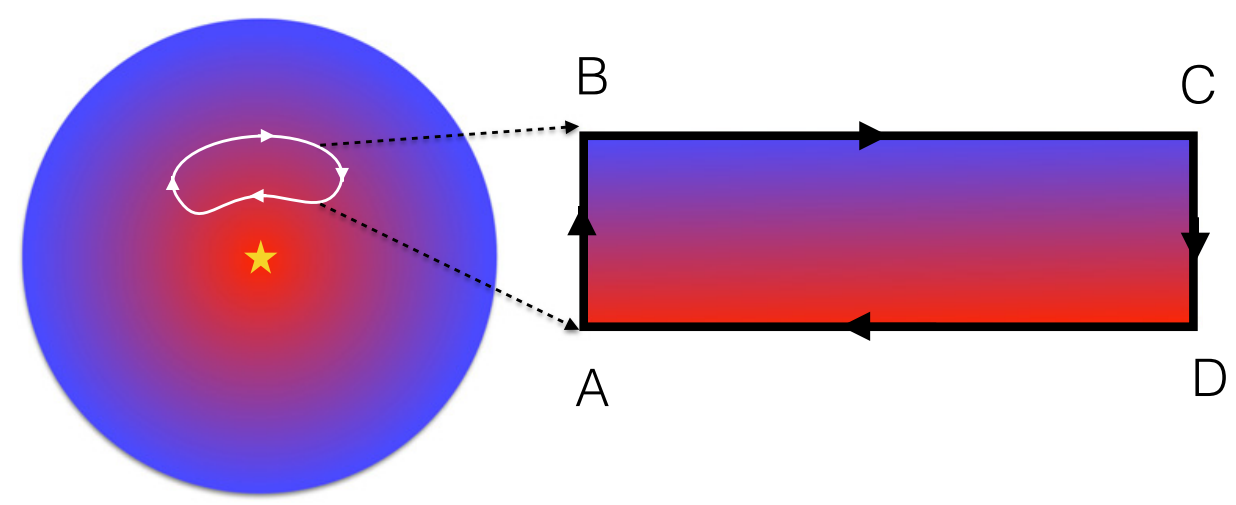

Fig. 2. Schematic illustration of the subcritical baroclinic instability. During its path along a vortex streamline (left panel), idealized as a rectangle (right panel), a fluid particle is buoyantly accelerated on the radial branches (A-B and C-D) and thermalized on the azimuthal branches (B-C and D-A). See text for details. Adapted from Lesur \& Papaloizou (2010).

Based on these numerical evidences, Lesur \& Papaloizou (2010) gave a simple physical interpretation to explain the growth of vortices induces by the SBI. Within an emerging vortex, which can be idealized as a rectangle such as shown on Figure 2, fluid particles are buoyantly accelerated on the two radial branches (thus the requirement $N_{R}^{2}<0$ ) and exchange heat on both horizontal branches (thus the requirement for a finite heat diffusion) so that they are thermalized when starting the next radial branch where they will be accelerated again, allowing the vortex to strengthen in the process.

In the past few years, these results have been independently confirmed (Lyra \& Klahr 2011; Raettig et al. 2013) and extended to more realistic geometries that include the disk density vertical stratification (Barge et al. 2016). Our knowledge of the SBI can be summarized as follows. First, it is severely suppressed in the presence of a magnetic field (Lyra \& Klahr 2011), so that it can only affect those regions of the disk known as the dead zone (see Sect. 2). The saturation of the SBI occurs through two mechanisms: the first involve centrifugal and parametric instabilities that destabilize the vortices themselves and prevent an unbounded growth (Lesur \& Papaloizou 2009). Being of small scale, they tend to be difficult to capture in numerical simulations. The second is through the emission of sound waves, modified by rotation, that take the form of spiral waves propagating radially away from the vortices. These spirals also have two consequences. First, they create an outward flux of angular momentum in the disk. It is customary in the literature to measure that flux using a dimensionless parameter called $\alpha^{1}$. In this

\footnotetext{
${ }^{1}$ There are several definitions of $\alpha$ in the literature, all of which are consistent to within a factor of a few. Observational constraints, based on typical evolutionary timescales of the various objects, suggest that $\alpha$ values typically range between $10^{-3}$ and a few times $10^{-1}$.
} 
particular case, $\alpha \sim$ a few times $10^{-3}$ (Lesur \& Papaloizou 2010; Raettig et al. 2013). Second, they induce a systematic and fast inward migration of vortices (Paardekooper et al. 2010), with can be considered to be a negative feedback on the saturation of the SBI. All these results, however, were established using very idealized simulations, so that the astrophysical implications of the SBI still remain to be investigated and quantified in more details.

\subsubsection{The convective over-stability}

The convective over-stability is an other flavour of the same process which has been discovered in axisymmetric 3D disks (Klahr \& Hubbard 2014). This instability is linear, and its growth rate can be estimated as

$$
\gamma \simeq-\Omega\left(\frac{N_{R}}{\Omega}\right)^{2} \frac{\Omega \tau_{c}}{1+\Omega^{2} \tau_{c}^{2}}
$$

from which we recover easily the two conditions for instability discussed above. The optimum growth rate is found when $\tau_{c}=1$ and assuming the disk is thin, it scales like $\Omega(H / R)^{2}$ which implies a relatively low growth rate in astrophysical applications.

Physically, it relies on the same phenomenology as the one discussed for the baroclinic instability (Fig. 2), except that fluid particles are not following a vortex streamline but are instead undergoing an epicyclic orbit. At each oscillation, fluid particles undergo a radial acceleration due to buoyancy, and cooling allows the flow to thermalize between two radial displacements. Averaged over one oscillation, this feedback loop leads to an amplification of epicyclic oscillations, hence the name of the instability (Latter 2016).

Overall this instability produces global horizontal oscillations, which if sufficiently amplified could break up into turbulence. Therefore, it has been suggested that the convective over-stability could act as a seed for the subcritical baroclinic instability (Lyra 2014). How this conclusion depends on $\tau_{c}$ and $N_{R} / \Omega$ however remains to be clarified.

\subsection{The vertical shear instability (VSI)}

Another consequence of PP disks thermal structure is the vertical shear instability (VSI), which is the disk analog of the Goldreich-Schubert-Fricke instability that has been familiar to the specialists of stellar dynamics since the 1960's (Goldreich \& Schubert 1967). By contrast, its potential relevance for accretion disks dynamics was only mentioned much later in the literature by Urpin \& Brandenburg (1998) and Urpin (2003), and the first numerical evidences for its existence have only been reported recently by Nelson et al. (2013) using global numerical simulations of locally isothermal disks (in which case the temperature $T$ depends only on the cylindrical radius $R$ ). As explained by Barker and Latter (2015), the VSI is fundamentally a centrifugal instability that relies on the existence of a vertical 

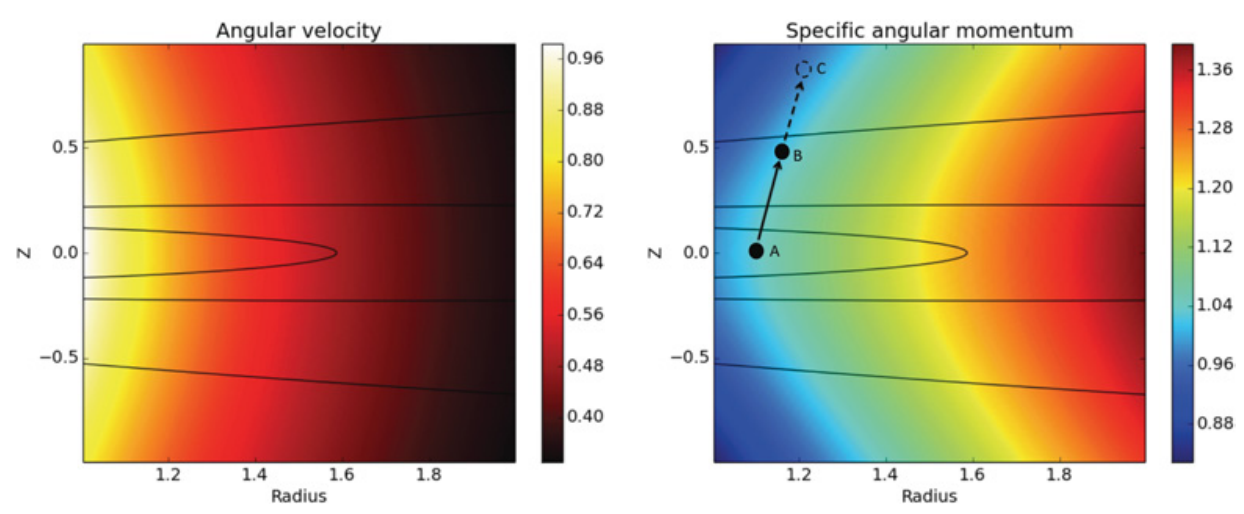

Fig. 3. Cartoon illustrating the physical mechanism of the Vertical Shear Instability (VSI). Angular velocity (resp. specific angular momentum) contours are shown on the left (resp. right) panel for a locally isothermal disk as a function of $R$ and $z$. On both panels, the disk density is shown using the solid contours, with levels equals to $0.5,0.1$ and $10^{-5}$ times the disk maximum density. A fluid particle, initially located in $A$, finds itself in $B$ with an excess angular momentum after a displacement along the black line (right panel) and keep moving upward and outward along the dashed line to $C$. See text for details.

gradient of $\Omega$, a well known consequence of baroclinicity in accretion disks. By taking the curl of the force balance relation, it is indeed easy to show that the baroclinic terms relates to the vertical gradient of $\Omega$ through:

$$
\frac{\partial R \Omega^{2}}{\partial R}=-\frac{1}{\rho^{2}}(\nabla \rho \times \nabla P) \cdot \boldsymbol{e}_{\boldsymbol{\varphi}}
$$

The vertical gradient of $\Omega$ (see also Fig. 3, left panel) implies that the second Solberg-Höiland criterion (3.2) now includes the term $\partial j / \partial z$, and this opens up the possibility of violating this criterion. Following Barker and Latter (2015), we illustrate such a possibility for the idealized and simple case of a locally isothermal disk and for vanishingly small cooling times $\tau_{c}$ for which buoyancy forces vanish. The contours of angular velocity and specific angular momentum are shown on Figure 3 for this model, respectively on the left and right hand side panels: consider the fluid particle initially in $\mathrm{A}$, with specific angular momentum $\mathcal{L}_{A}$. If it is displaced along the black line, it will find itself in position B with an excess angular momentum (because $\mathcal{L}_{A}>\mathcal{L}_{B}$ ), in clear violation of the Rayleigh criterion, and will keep moving upward and outward.

In real disks, however, the vertical entropy profiles tends to be stabilizing. Buoyancy forces oppose the motions just described, and some amount of thermal diffusion (or radiative cooling) is necessary to prevent a complete stabilization of the VSI (which materializes in an adiabatic, or isentropic, disk by the vertical gradient of $\Omega$ vanishing). Once again, the disk thermal properties turn out to be 

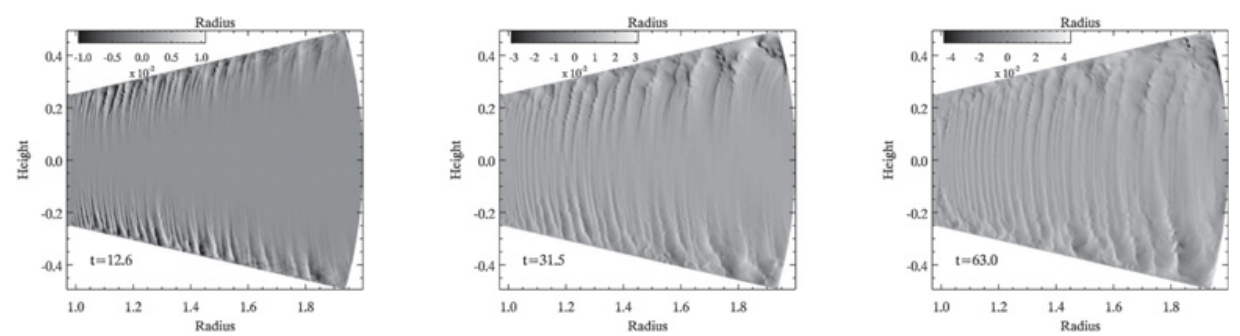

Fig. 4. Vertical velocity perturbations snapshots in the $(R, z)$ plane in a global simulation of a locally isothermal disk during the growth of the vertical shear instability. Time increases from left to right. From Nelson et al. (2013).

a key ingredient of the process! In addition, viscosity also stabilizes the smallest unstable scales (as shown early on by Nelson et al. 2013). For these reasons, the VSI is in fact a double-diffusive instability (Barker and Latter 2015): it develops at intermediate scales for which neither viscosity nor buoyancy dominate the fluid motions. Lin \& Youdin (2015) have recently coupled these simple arguments with analytical models of PP disks to show that the VSI should be expected to develop at radii between a few AUs and $\sim 100$ AUs thanks to sufficiently short cooling times at those locations.

The simple physical interpretation of the VSI discussed above should not hide the complexity of the linear modes of the instability, which have been the subject of detailed calculations in the recent literature (Nelson et al. 2013; Barker and Latter 2015; Umurhan et al. 2016a). These modes come in two flavors: the first type is composed of inertial waves that are destabilized by the vertical angular velocity gradient. They have modest growth rates and grow at relatively long wavelengths. The second type is composed of surface modes that grow faster and at smaller wavelengths set by the flow kinematic viscosity. For these reasons, they are more difficult to capture in numerical simulations, and are likely to be affected by numerical effects in the simulations that have been published so far. For this reason, it is still presently unclear which class of modes will dominate in disks during the nonlinear evolution of the VSI, and current simulations must be interpreted with care.

Despite these concerns, a number of global numerical simulations have been published that investigate the nonlinear outcome of the VSI. Nelson et al. (2013) showed that the VSI manifests itself as short radial wavelengths perturbations (with comparatively larger vertical scale) that first develop in the disk upper layers before eventually affecting the entire disk (Fig. 4). Turbulence ensues, and lead to angular momentum transport rates that depends on the disk cooling time. An upper bound of $\alpha \sim 10^{-3}$ is found in the locally isothermal limit for which $N_{z}^{2}$ vanishes (Nelson et al. 2013), while values ranging from a few times $10^{-5}$ to a few times $10^{-4}$ are found for finite values of the cooling time (Richard et al. 2016). In this situation, the VSI appears to saturate via the triggering of short lived 
SBI-unstable vortices. This is not unexpected, since the conditions of existence for the VSI and the SBI are relatively similar, and suggest that the VSI could act as a seed for the SBI. Finally, we note that more realistic simulations that include heating by the central star and radiative cooling by dust particles are now feasible (Stoll \& Kley 2014, 2016): the typical $\alpha \sim$ a few times $10^{-4}$ that have been reported are in agreement with the idealized simulations described above.

\section{Alternative routes to turbulence}

At this stage, it should be clear that the complexity of accretion disks is such that several physical processes have the potential to affect their dynamics. The reader should thus not be surprised to learn that several other possibilities have been proposed in the literature that might lead to angular momentum transport in disks. Some of them apply only in special situations (for example, the gravitational instability requires that the mass of the disk itself be significant), while other have only been very recently proposed and must be investigated more thoroughly. In the present section, we briefly review those of them that are the most intensively discussed in the literature. We omit on purpose a detailed discussion of the Rossby Wave Instability (RWI, Lovelace et al. 1999). Although it may well be important for some aspects of accretion disks dynamics and observations, the RWI is confined to the vicinity of vortensity extrema and unlikely to have a global impact on angular momentum transport in disks.

\subsection{The gravitational instability (GI)}

So far, we restricted the discussion to massless accretion disk. However, the disk self-gravity can render the flow unstable to a gravitational instability (GI) in massive disks and could have some implications for the dynamics of PP disks and for that of AGN disks. The properties of GIs in disks have been much investigated, and we point the interested reader to the recent reviews by Kratter \& Lodato (2016) for the case of PP disks, and by Lodato (2012) for the case of AGN disks. Here, we briefly recall the main points, focusing of those aspects of the problem that are relevant for angular momentum transport. The importance of self-gravity is measured using the Toomre parameter (Toomre 1964):

$$
Q=\frac{c_{s} \kappa}{\pi G \Sigma} \sim\left(\frac{H}{R}\right)\left(\frac{M}{M_{d}}\right),
$$

where $G$ is the gravitational constant, $c_{s}$ is the speed of sound and $\Sigma$ is the disk surface density. Accretion disks are linearly unstable to axisymmetric perturbations when $Q$ is smaller than unity and linearly unstable to non-axisymmetric perturbations when $Q$ is of order unity. The second relation in Equation (6.1) above, in which $M_{d}$ stands for the disk mass contained within a radius $\mathrm{R}$, shows that this situation arises when the enclosed disk mass with a radius $R$ is of order $(H / R) M$. For this reasons, GIs are expected to develop preferentially in disks outer parts and are unlikely to explain angular momentum transport in disks inner parts. 
While these linear arguments are fairly simple and well established, understanding how GIs saturate is much more complicated. As shown by Equation (6.1), $Q$ depends on the disk temperature through $c_{s}$, so that the outcome of the instability depends once more on the disk heating and cooling processes. For AGN disks that are not irradiated, heating results from the thermalization of the kinetic energy of the gas motions generated by the GI itself. The saturation of the instability depends on the cooling timescale $\tau_{c}$ in the sense that the disk reaches a quasi steady state in which heating and cooling balance each other (Paczynski 1978). The rate of angular momentum transport in that case was numerically found to be (Gammie 2001):

$$
\alpha \sim \frac{4}{9 \gamma(\gamma-1) \Omega \tau_{c}}
$$

This result is only marginally modified when irradiation is important in the energy budget (as is the case for PP disks), as shown by means of idealized 2D simulations by Rice et al. (2011). When $\tau_{c}=10 \Omega^{-1}$ (such as is typical of PP disks midplanes), the above formula gives $\alpha \sim 10^{-2}$, thereby possibly accounting for angular momentum transport in disks outer parts provided the disk mass is large enough. For short cooling timescales, $\alpha$ can reach large values, and it has been found early on in this case that disks can fragment and form bound structures (Gammie 2001). This has been interpreted as a possible route to form planets in $\mathrm{PP}$ disks, although the issue is widely debated in the literature and depends on subtle numerical issues (Kratter \& Lodato 2016).

\subsection{Vertical convection}

As mentioned in Section 3, accretion disk can become convectively unstable to vertical convection if $N_{z}^{2}<0$ and this has historically been considered as a possible avenue toward angular momentum transport (Vila 1978; Lin \& Papaloizou 1980). While early simulations suggested that angular momentum transport was directed inward rather than outward (Kley et al. 1993; Stone \& Balbus 1996), this surprising result was more recently shown to be due to the Rayleigh number $R a$, which compares the relative importance of buoyancy and dissipative processes, being too small. At large Rayleigh number (such as found in accretion disks), outward transport is recovered, with typical $\alpha$ values of order a few times $10^{-4}$ (Lesur and Ogilvie 2010). All these results, however, leave aside the question of the maintenance of a negative vertical entropy gradient in the disk, which requires a source of heating in the disk midplane region.

Recently, Bodo et al. (2012) and Hirose et al. (2014) both suggested, by means of MHD simulations, that such a heating could be provided by MRI turbulence. Although more work is needed to assess its astrophysical implications, these results open the possibility for an interesting coupling between the MRI and convection that would affect the dynamics of erupting disks, such as in dwarf novae and/or soft X-ray transients. 


\subsection{The Zombie vortex instability (ZVI)}

The ZVI was first identified in anelastic simulations (Barranco \& Marcus 2005). This non-linear instability appears in vertically stratified rotating sheared flows which are stable for convection (i.e., $N_{z}^{2}>0$ ). The presence of vertical buoyancy implies that in addition to epicyclic modes, buoyancy modes are also present in the system. As shown by Marcus et al. (2013), an isolated vortex in such a flow spontaneously triggers non-axisymmetric buoyancy waves which eventually break in critical layers. Marcus et al. (2013) proposed that these critical layers were in turn unstable and could generate new vortices, restarting the cycle and filling the entire domain with vortices.

This process was observed both in Boussinesq (Marcus et al. 2013) and compressible simulations (Marcus et al. 2015), and found to be linearly associated with unstable buoyancy waves (Umurhan et al. 2016b). However, critical layers being singularities of the linear inviscid equations, they are necessarily regularized by viscosity and non-linear effects. As expected, the ZVI is therefore highly sensitive to the diffusion operator used in numerical simulations, and seems to be inexistent when second order dissipation is used to model the flow. In addition, thermal diffusion must be sufficiently small to avoid the damping of buoyancy waves required by this instability (Lesur \& Latter 2016). Whether the ZVI is applicable to PP disks is therefore a very open debate.

\subsection{The stratorotational instability (SRI)}

The SRI occurs in situations similar to the one of the ZVI: rotating shear flow with a stable vertical stratification, except that it requires some sort of reflecting boundary condition in the radial direction. It is a linear instability, first identified in Taylor-Couette flows (Yavneh et al. 2001). The instability is primarily due to a resonance between Kelvin waves which are traveling along the radial boundaries (walls). For this resonance to be effective, the walls have to be sufficiently close. The linear analysis shows that unstable modes have a vertical wavelength $\lambda_{z}$ correlated to the wall separation:

$$
\lambda_{z} \sim \frac{\Omega}{N_{z}} L_{\mathrm{wall}}
$$

where $L_{\text {wall }}$ is the radial distance between the walls (Umurhan 2006). Since PP disks always have $N_{z} \lesssim \Omega$ (Dubrulle et al. 2005), the stratorotational instability can only grow when $L_{\text {wall }}<H$.

With an astrophysical application in mind, it is possible to generalize the notion of rigid walls by assuming that a low density parcel of gas is radially sandwiched between two parcels of high density gas. In this case, the stratorotational instability still shows up, but the required $L_{\text {wall }}$ then decreases as the density contrast decreases, leading to the suppression of the instability when the parcels have equal densities (Lesur 2007, Fig. 68).

Overall, these results suggest that the SRI might appear in those regions of accretion disks where the flow is radially confined, such as narrow gaps that are 
created by a planet, but that it is not a robust way to transport angular momentum in the entire disk.

\subsection{The Papaloizou-Pringle instability (PPI)}

Discovered by Papaloizou \& Pringle (1984), the PPI relies on a resonance between spiral density waves exchanging energy accross the corotation radius of the disk (Goldreich et al. 1986). Like the SRI, it depends on the presence of radial boundaries in the disk, so that it is equally unlikely to be a robust mechanism for the radial transport of angular momentum in disks. For more details on the physical mechanism of the PPI, we refer the interested reader to the review article of Papaloizou \& Lin (1995).

\section{Conclusion}

The length and diversity of this review shows that the research community is not short of ideas to account for angular momentum transport in Keplerian shear flows via turbulence, even within the somewhat restrictive framework of hydrodynamics. Some of the proposed solutions (like the subcritical transition - see Sect. 4) are heavily inspired from the traditional field of hydrodynamics, while some others (vertical shear instability, baroclinic instability, or gravitational instability) exploit some of the peculiar properties of the astrophysical objects themselves. However, at present time, it is fair to say that none of them (including the MRI) appears to hold the promise of explaining - alone - how angular momentum is transported outward in accretion disks. It is likely that the later results from a combination of several of these processes that operate simultaneously or at different locations in disks. For example, in PP disks, the most recent results suggest to combine the MRI, likely modified by the Hall term, and the VSI (which might excite the SBI, as discussed in Sect. 5.3), with GIs and/or infall onto the disk (Lesur et al. 2015) possibly playing a role in the outer parts. In view of this complexity, direct observations of turbulence in PP disks, now starting to be feasible with ALMA, will be of paramount importance. Ironically, the first such observations suggest very low levels of turbulence (Flaherty et al. 2015; Pinte et al. 2016), incompatible with any of the mechanisms discussed in this review as well as with our current understanding of the MRI.

At the more fundamental level, the reason for the apparent hydrodynamic stability of Keplerian flows remains elusive today. This is particularly surprising given the huge reservoir of free energy that is available and must be understood. Progress will have to come simultaneously from all sides of the battlefront, be it experimental, numerical or theoretical, as each one suffer from its own limitations. For example, experiments less sensitive to the end caps, such as proposed by Leclercq et al. (2016), must be repeated at larger Reynolds numbers. They will have to be complemented by better resolved direct numerical simulations that will allow, thanks to ever increasing computational progress, higher $R e$ to be explored. Theoretical work is also needed, and should take advantage, as discussed 
in Section 4, of the rapidly expanding literature devoted to the question of shear flow stability in the hydrodynamic community (see for example the review of Eckhardt et al. 2007 on the transition to turbulence in pipe flows). Another avenue which could be explored more systematically is the sensitivity of Keplerian flows to external perturbations. Even if accretion disks are nonlinearly stable, they might be subject to turbulence triggered by low amplitude motions in the disk environment which are subsequently amplified (Ioannou \& Kakouris 2001). Only a combination of such widely different approaches will help settle the question of whether or not Keplerian flows are vulnerable to a subcritical transition to turbulence that Jean-Paul Zahn tried to tackle 20 years ago.

The authors warmly acknowledge Adrian Barker \& Henrik Latter for many discussions related to the physical processes presented in this paper.

\section{References}

Avila, M., 2012, Phys. Rev. Lett., 108(12), 124501

Bai, X.-N., 2014, ApJ, 791, 137

Bai, X.-N., 2015, ApJ, 798, 84

Bai, X.-N., 2016, ApJ, 821, 80

Bai, X.-N., \& Stone, J.M., 2013, ApJ, 767, 30

Balbus, S.A., \& Hawley, J.F., 1991, ApJ, 376, 214

Balbus, S.A., \& Hawley, J.F., 1998, Rev. Mod. Phys., 70, 1

Balbus, S.A., Hawley, J.F., \& Stone, J.M., 1996, ApJ, 467, 76

Barge, P., Richard, S., \& Le Dizès, S., 2016, A\&A, 592, A136

Barker, A.J., \& Latter, H.N., 2015, MNRAS, 450, 21

Barranco, J.A., \& Marcus, P.S., 2005, ApJ, 623, 1157

Bodo, G., Cattaneo, F., Mignone, A., \& Rossi, P., 2012, ApJ, 761, 116

Chandrasekhar, S., 1961, Hydrodynamic and Hydromagnetic Stability

Chiang, E.I., \& Goldreich, P., 1997, ApJ, 490, 368

D’Alessio, P., Cantö, J., Calvet, N., \& Lizano, S., 1998, ApJ, 500, 411

Dubrulle, B., Marié, L., Normand, C., Richard, D., Hersant, F., \& Zahn, J.-P., 2005, A\&A, 429, 1

Dullemond, C.P., Hollenbach, D., Kamp, I., \& D'Alessio, P., 2007, Protostars and Planets V, p. 555

Eckhardt, B., Schneider, T.M., Hof, B., \& Westerweel, J., 2007, Ann. Rev. Fluid Mech., 39,447

Edlund, E.M., \& Ji, H., 2014, Phys. Rev. E., 89(2), 021004

Flaherty, K.M., Hughes, A.M., Rosenfeld, K.A., Andrews, S.M., Chiang, E., Simon, J.B., Kerzner, S., \& Wilner, D.J., 2015, ApJ, 813, 99

Fleming, T., \& Stone, J.M., 2003, ApJ, 585, 908

Frank, A., Ray, T.P., Cabrit, S., Hartigan, P., Arce, H.G., Bacciotti, F., Bally, J., Benisty, M., Eislöffel, J., Güdel, M., Lebedev, S., Nisini, B., \& Raga, A., 2014, Protostars and Planets VI -1, p. 451 
Frank, J., King, A.R., \& Raine, D.J., 1985, Accretion power in astrophysics

Fromang, S., Latter, H., Lesur, G., \& Ogilvie, G.I., 2013, A\&A, 552, A71

Gammie, C.F., 1996, ApJ, 457, 355

Gammie, C.F., 2001, ApJ, 553, 174

Goldreich, P., Goodman, J., \& Narayan, R, 1986, MNRAS, 221, 339

Goldreich, P., \& Lynden-Bell, D., 1965, MNRAS, 130, 125

Goldreich, P., \& Schubert, G., 1967, ApJ, 150, 571

Grossmann, S., Lohse, D., \& Sun, C., 2016, Ann. Rev. Fluid Mech., 48, 53

Hawley, J.F., Balbus, S.A., \& Winters, W.F., 1999, ApJ, 518, 394

Heinemann, T., \& Papaloizou, J.C.B., 2009, MNRAS, 397, 52

Hirose, S., Blaes, O., Krolik, J.H., Coleman, M.S.B., \& Sano, T., 2014, ApJ, 787, 1

Ioannou, P.J., \& Kakouris, A., 2001, ApJ, 550, 931

Ji, H., Burin, M., Schartman, E., \& Goodman, J., 2006, Nature, 444, 343

Johnson, B.M., \& Gammie, C.F., 2005, ApJ, 626, 978

Klahr, H., 2004, ApJ, 606, 1070

Klahr, H., \& Hubbard, A., 2014, ApJ, 788, 21

Klahr, H.H., \& Bodenheimer, P., 2003, ApJ, 582, 869

Kley, W., Papaloizou, J.C.B., \& Lin, D.N.C., 1993, ApJ, 416, 679

Kratter, K., \& Lodato, G., 2016, ARA\&A, 54, 271

Kunz, M.W., \& Lesur, G., 2013, MNRAS, 434, 2295

Latter, H.N., 2016, MNRAS, 455, 2608

Leclercq, C., Partridge, J.L., Augier, P., Dalziel, S.B., \& Kerswell, R.R., 2016, J. Fluid Mech. 791, 608

Lesur, G., 2007, Ph.D. thesis, Université Joseph-Fourier - Grenoble I, https://tel. archives-ouvertes.fr/tel-00166016

Lesur, G., Hennebelle, P., \& Fromang, S., 2015, A\&A, 582, L9

Lesur, G., Kunz, M.W., \& Fromang, S., 2014, A\&A, 566, A56

Lesur, G., \& Longaretti, P.-Y., 2005, A\&A, 444, 25

Lesur, G., \& Ogilvie, G.I., 2010, MNRAS, 404, L64

Lesur, G., \& Papaloizou, J.C.B., 2009, A\&A, 498, 1

Lesur, G., \& Papaloizou, J.C.B., 2010, A\&A, 513, A60

Lesur, G.R.J., \& Latter, H., 2016, MNRAS, 462, 4549

Lin, D.N.C., \& Papaloizou, J., 1980, MNRAS, 191, 37

Lin, M.-K., \& Youdin, A.N., 2015, ApJ, 811, 17

Lodato, G., 2012, Adv. Astron., 2012, 846875

Lopez, J.M., \& Avila, M., 2017, J. Fluid Mech., 817, 21

Lovelace, R.V.E., Li, H., Colgate, S.A., \& Nelson, A.F., 1999, ApJ, 513, 805

Lyra, W., 2014, ApJ, 789, 77

Lyra, W., \& Klahr, H., 2011, A\&A, 527, A138

Marcus, P.S., Pei, S., Jiang, C.-H., Barranco, J.A., Hassanzadeh, P., \& Lecoanet, D., 2015, ApJ, 808, 87

Marcus, P.S., Pei, S., Jiang, C.-H., \& Hassanzadeh, P., 2013, Phys. Rev. Lett., 111, 084501 
Nelson, R.P., Gressel, O., \& Umurhan, O.M., 2013, MNRAS, 435, 2610

Ostilla-Mónico, R., Verzicco, R., Grossmann, S., \& Lohse, D., 2014, J. Fluid Mech., 748, R3

Paardekooper, S.-J., Lesur, G., \& Papaloizou, J.C.B., 2010, ApJ, 725, 146

Paczynski, B., 1978, AcTa, 28, 91

Paoletti, M.S., \& Lathrop, D.P., 2011, Phys. Rev. Lett., 106(2), 024501

Paoletti, M.S., van Gils, D.P.M., Dubrulle, B., Sun, C., Lohse, D., \& Lathrop, D.P., 2012, A\&A, 547, A64

Papaloizou, J.C.B., \& Lin, D.N.C., 1995, ARA\&A, 33, 505

Papaloizou, J.C.B., \& Pringle, J.E., 1984, MNRAS, 208, 721

Petersen, M.R., Julien, K., \& Stewart, G.R., 2007a, ApJ, 658, 1236

Petersen, M.R., Stewart, G.R., \& Julien, K., 2007b, ApJ, 658, 1252

Pinte, C., Dent, W.R.F., Ménard, F., Hales, A., Hill, T., Cortes, P., \& de GregorioMonsalvo, I., 2016, ApJ, 816, 25

Quataert, E., 2001, in B.M. Peterson, R.W. Pogge, \& R.S., Polidan (eds.), Probing the Physics of Active Galactic Nuclei, Vol. 224 of Astronomical Society of the Pacific Conference Series, p. 71

Raettig, N., Lyra, W., \& Klahr, H., 2013, ApJ, 765, 115

Rice, W.K.M., Armitage, P.J., Mamatsashvili, G.R., Lodato, G., \& Clarke, C.J., 2011, MNRAS, 418, 1356

Richard, D., 2001, Ph.D. thesis, Observatoire de Paris GIT/SPEC CEA Saclay

Richard, D., \& Zahn, J.-P., 1999, A\&A, 347, 734

Richard, S., Nelson, R.P., \& Umurhan, O.M., 2016, MNRAS, 456, 3571

Rincon, F., Ogilvie, G.I., \& Cossu, C., 2007, A\&A, 463, 817

Schartman, E., Ji, H., Burin, M.J., \& Goodman, J., 2012, A\&A, 543, A94

Shi, L., Hof, B., Rampp, M., \& Avila, M., 2017, Phys. Fluids, 29(4), 044107

Stoll, M.H.R., \& Kley, W., 2014, A\&A, 572, A77

Stoll, M.H.R., \& Kley, W., 2016, A\&A, 594, A57

Stone, J.M., \& Balbus, S.A., 1996, ApJ, 464, 364

Suzuki, T.K., \& Inutsuka, S.-i., 2009, ApJ, 691, L49

Suzuki, T.K., Muto, T., \& Inutsuka, S.-i., 2010, ApJ, 718, 1289

Tassoul, J.-L., 2007, Stellar Rotation

Taylor, G.I., 1923, Philos. Trans. R. Soc. London Ser. A, 223, 289

Tevzadze, A.G., Chagelishvili, G.D., Zahn, J.-P., Chanishvili, R.G., \& Lominadze, J.G., 2003, A\&A, 407, 779

Tillmark, N., \& Alfredsson, P.H., 1992, J. Fluid Mech., 235, 89

Toomre, A., 1964, ApJ, 139, 1217

Turner, N.J., Fromang, S., Gammie, C., Klahr, H., Lesur, G., Wardle, M., \& Bai, X.-N., 2014, Protostars and Planets VI, p. 411

Umurhan, O.M., 2006, MNRAS, 365, 85

Umurhan, O.M., Nelson, R.P., \& Gressel, O., 2016a, A\&A, 586, A33

Umurhan, O.M., Shariff, K., \& Cuzzi, J.N., 2016b, ApJ, 830, 95

Urpin, V., 2003, A\&A, 404, 397 
Urpin, V., \& Brandenburg, A., 1998, MNRAS, 294, 399

van Gils, D.P.M., Bruggert, G.-W., Lathrop, D.P., Sun, C., \& Lohse, D., 2011, Rev. Sci. Instrum., 82(2), 025105

Velikhov, E.P., 1959, Sov. Phys. JETP 36, 995

Vila, S.C., 1978, ApJ, 223, 979

Williams, J.P., \& Cieza, L.A., 2011, ARA\&A, 49, 67

Woitke, P., Kamp, I., \& Thi, W.-F., 2009, A\&A, 501, 383

Yavneh, I., McWilliams, J.C., \& Jeroen M.M., 2001, J. Fluid Mech., 448, 1 